\title{
Using concept mapping to learn about A level physics students' understandings of particle physics
}

\author{
Helen Gourlay \\ ${ }^{1}$ STEM Education Research Group, Department of Education, Brunel University London, Kingston Lane, \\ Uxbridge, UB8 $3 \mathrm{PH}$
}

\begin{abstract}
This article describes a small-scale piece of research using concept mapping to elicit A level students' understandings of particle physics, which was presented in the Special Session on Physics Education and Outreach at ICNFP 2017. Fifty-nine Year 12 (16- and 17-year-old) students from two London schools participated in the research. The exercise took place during school physics lessons. Students were shown how to make a concept map and were provided with topic-specific key words. Their concept maps were analysed by identifying the knowledge propositions the students had represented, and comparing these with propositions developed from the examination specification they were studying. The only correct statement made by most of the students in both schools was that annihilation takes place when matter and antimatter collide, although some students may have been unable to distinguish between annihilation and pair production. A high proportion of students knew of up, down and strange quarks, and that the electron is a lepton. However, some students appeared to have a misconception that everything is made of quarks. Students found it harder to classify tau particles than they did electrons and muons. Where students made incorrect links about muons and tau particles their concept maps suggested that they thought they were mesons or quarks.
\end{abstract}

\section{Introduction}

Before working in university partnership initial teacher education I worked in schools as a teacher of science and physics. I taught the particle physics topic at A level from 2004 onwards. In common with many colleagues, I had not studied particle physics at A level, nor in my undergraduate degree: Particle physics became a compulsory part of A level courses in England only in 2008. That said, it had been taught in some syllabuses since the 1990s [1]. At that time, concern had been expressed about whether or not particle physics could be taught in a meaningful way prior to the study of quantum mechanics at undergraduate level [2].

I was fortunate to have the opportunity to visit CERN, funded by the National Science Learning Centre (now STEM Learning), which contributed to my growing interest in, and enthusiasm for the topic. It was a particularly exciting time because there was much anticipation about the possibility of observing the Higg's boson for the first time. My A level students responded well to the topic, being keen to learn about an active area of science research.

I came across the method of concept mapping in material for teaching pre-service science teachers about children's thinking and learning in science. This material adopted a constructivist model 
of learning, i.e. that learners develop mental frameworks in which new knowledge builds upon their existing ideas. Concept maps had been developed as a means of uncovering learners' cognitive structures [3]. It was suggested that the following are key features of quality in concept maps produced by learners: A hierarchical structure with more general concepts at the top and more specific ones at the bottom; labelled links showing the relationship between concepts; cross-linking between different areas of the topic; and the inclusion of examples.

There is a body of science education research, which tells us that children come to science lessons with naive ideas about science phenomena [4]. These preconceptions are often at odds with accepted scientific theory and may be hard to shift. Research into children's ideas about particle theory has focused on material at the atomic level - broadly situated within the subject discipline of chemistry. This perspective is exemplified by the following definition:

The key understanding of the matter concept is the appreciation that all things in the universe consist of matter and, although they can change in form and composition, the total mass remains the same. Further, all the changes and properties can be explained by the molecular and atomic structure theories of matter [5].

The particle physics topic at A level rests on a different assumption. A paradigm shift has taken place in scientific understanding: Conservation of mass does not necessarily apply - there is only conservation of energy in accordance with Einstein's equation $E=m c^{2}$ (where the total energy includes rest-mass energy). In this topic, the atom is not the basic unit, but rather it is a special case - composed of only three of the particles of physics (being up and down quarks, and electrons).

Little research has been conducted investigating children's ideas about the subatomic level. I was able only to find relevant material relating to understanding of protons, neutrons and electrons. This research suggested that children had difficulty in remembering the charges on these particles, and that this may explain their lack of understanding of the electrostatic force of attraction between the electron and the nucleus [5] [6].

I was interested to know what A level students had learned or not learned when moving on to look in even finer detail at the subatomic level, and hence my research question was: What does concept mapping tell us about A level students' understandings of the particle physics topic?

\section{Methods}

Two state schools were recruited from the university partnership (schools working with our preservice teachers). Both were located in outer London and the students who opted to participate were in Year 12 (aged 16 and 17 years). One was a mixed comprehensive school and the other was a selective boys' school. Students in both schools had studied particle physics in the AQA specification [7] and had already been examined on it as part of their course. 59 students took part.

The concept mapping activity took place during the course of a lesson (a period of about one hour). Students were first introduced to concept mapping using examples taken from White and Gunstone [8], in which pupils had mapped the rock cycle (a topic which the A level students would have studied previously). The key features of high quality concept maps, given above, were discussed in the context of these examples.

I had prepared a list of concept words chosen from a commonly used A level textbook [9]. I had piloted the list with my group of preservice physics teachers, which led to the following concepts being used for the study: annihilation; bottom; baryon; antiparticle; atom; electron; down; neutrino; charm; hadron; meson; nucleon; lepton; matter; muon; proton; neutron; top; nucleus; quark; up; particle; tau; strange. 
Participants were given instructions for creating a concept map based on those given by White and Gunstone[8]. They wrote the concept words on sticky notes so that they could move them around on paper during the process. Concept maps presented below have been reproduced using CMap Tools software for anonymization purposes.

To analyse the concept maps, I first wrote down each of the knowledge propositions that participants had represented on their maps. Using the exam specification they had studied, and applying professional knowledge from teaching the topic in the past, I constructed a list of knowledge propositions that students might have been expected to know. I then compared the two, noting the numbers of correct propositions, as well as those that might be considered to be incorrect, or to indicate a misconception or misunderstanding.

\section{Results}

\subsection{Correct propositions}

Table 1 shows the propositions that were most frequently represented in students' concept maps. Propositions shown by 10 per cent of the students or more ( 6 or more students) have been included [10].

An example of a students' concept map is shown in figure 1 [10]. This concept map exemplifies many of the most common correctly represented ideas. 42 of 59 students correctly showed that up, down and strange particles are quarks, and 32 identified the electron as a lepton. More than half the students showed that mesons and neutrinos are leptons. 31 students showed that baryons and mesons are hadrons. Note that the student whose concept map is shown in figure 1 made an error in suggesting that hadrons are made of 3 quarks. This was not a common error, as 25 students' concept maps correctly showed that baryons are made of 3 quarks. However, fewer than half the students noted the quark-antiquark structure of mesons.

Although 36 students showed that when a particle and the corresponding antiparticle meet they annihilate each other, only 13 represented what I had expected, which was that photons are produced. And there seemed to be some confusion about annihilation and pair production, exemplified by some of the statements I considered not to be wholly correct:

- Some leptons are produced by annihilation

- Annihilation produces matter and antimatter

- Pair production is when an electron with too much energy produces photons

- Pair production is when a photon with sufficient energy makes two particles that repel each other

- Pair production is what happens when a particle splits in two

- Annihilation produces hadrons and leptons

- Quarks can annihilate to produce bigger, heavier, more exotic particles as well as photons

There is a possibility that the some of these statements indicates knowledge going beyond the A level syllabus, since (for example) the top quark may be produced in collisions between matter and antimatter. However, the students' concept maps did not give enough information for me to be certain.

\subsection{Misunderstandings}

In total, the students produced 220 knowledge propositions, of which I considered 70 to show misunderstandings. Because few students made identical errors, I grouped them into categories. The lepton 
Table 1. Correct propositions most frequently represented by students

\begin{tabular}{|c|c|c|c|}
\hline Proposition & $\begin{array}{l}\text { No. students } \\
\text { school } 1 \\
(\mathrm{n}=21)\end{array}$ & $\begin{array}{l}\text { No. students } \\
\text { school } 2 \\
(\mathrm{n}=38)\end{array}$ & $\begin{array}{l}\text { Total no. } \\
\text { of students } \\
(\mathrm{n}=59)\end{array}$ \\
\hline Up is a type of quark & 10 & 33 & 43 \\
\hline Down is a type of quark & 10 & 33 & 43 \\
\hline Strange is a type of quark & 10 & 32 & 42 \\
\hline When a particle and its antiparticle & 13 & 23 & 36 \\
\hline Electron is a type of lepton & 6 & 26 & 32 \\
\hline Baryon is a type of hadron & 3 & 28 & 31 \\
\hline Meson is a type of hadron & 3 & 28 & 31 \\
\hline Proton is a type of baryon & 5 & 21 & 26 \\
\hline Neutron is a type of baryon & 5 & 21 & 26 \\
\hline Baryons are made of three quarks & 7 & 18 & 25 \\
\hline Muon is a type of lepton & 5 & 20 & 25 \\
\hline Neutrino is a type of lepton & 6 & 18 & 24 \\
\hline $\begin{array}{l}\text { Mesons are made of a quark and antiquark } \\
\text { pair }\end{array}$ & 6 & 16 & 22 \\
\hline $\begin{array}{l}\text { The proton is a constituent of the atom } \\
\text { (or nucleus) }\end{array}$ & 4 & 12 & 16 \\
\hline $\begin{array}{l}\text { The neutron is a constituent of the atom } \\
\text { (or nucleus) }\end{array}$ & 4 & 12 & 16 \\
\hline $\begin{array}{l}\text { When annihilation takes places the mass } \\
\text { of the particles is converted into energy in } \\
\text { the form of photons }\end{array}$ & 5 & 8 & 13 \\
\hline Mesons are made of two quarks & 6 & 5 & 11 \\
\hline A neutron is made of udd quarks & 2 & 6 & 8 \\
\hline $\begin{array}{l}\text { An antiparticle has the opposite charge to the } \\
\text { corresponding particle }\end{array}$ & 3 & 4 & 7 \\
\hline Hadrons are subject to the strong nuclear force & 3 & 3 & 6 \\
\hline The proton is the only stable baryon & 2 & 4 & 6 \\
\hline Leptons are subject to the weak interaction & 4 & 2 & 6 \\
\hline A proton is made of uud quarks & 0 & 6 & 6 \\
\hline
\end{tabular}

category had the most errors, as shown in table 2. The most common errors were in classification of the muon and tau particles. An example of this type of error is shown in figure 2, where the student has classified them as mesons. Note that the four students classified the tau particle as a quark.

Students also represented a number of propositions that showed misunderstandings about fundamental particles:

- Antiparticles are made of antiquarks

- Quarks and baryons are elementary particles

- Leptons are made of quarks

- Matter and particles are made up of atoms which are made up of quarks

- Particles are made of quarks 
- Atom is the fundamental particle of all matter

- Electrons are made of quarks

- Everything is made of quarks

- Particles and antiparticles are made of quarks

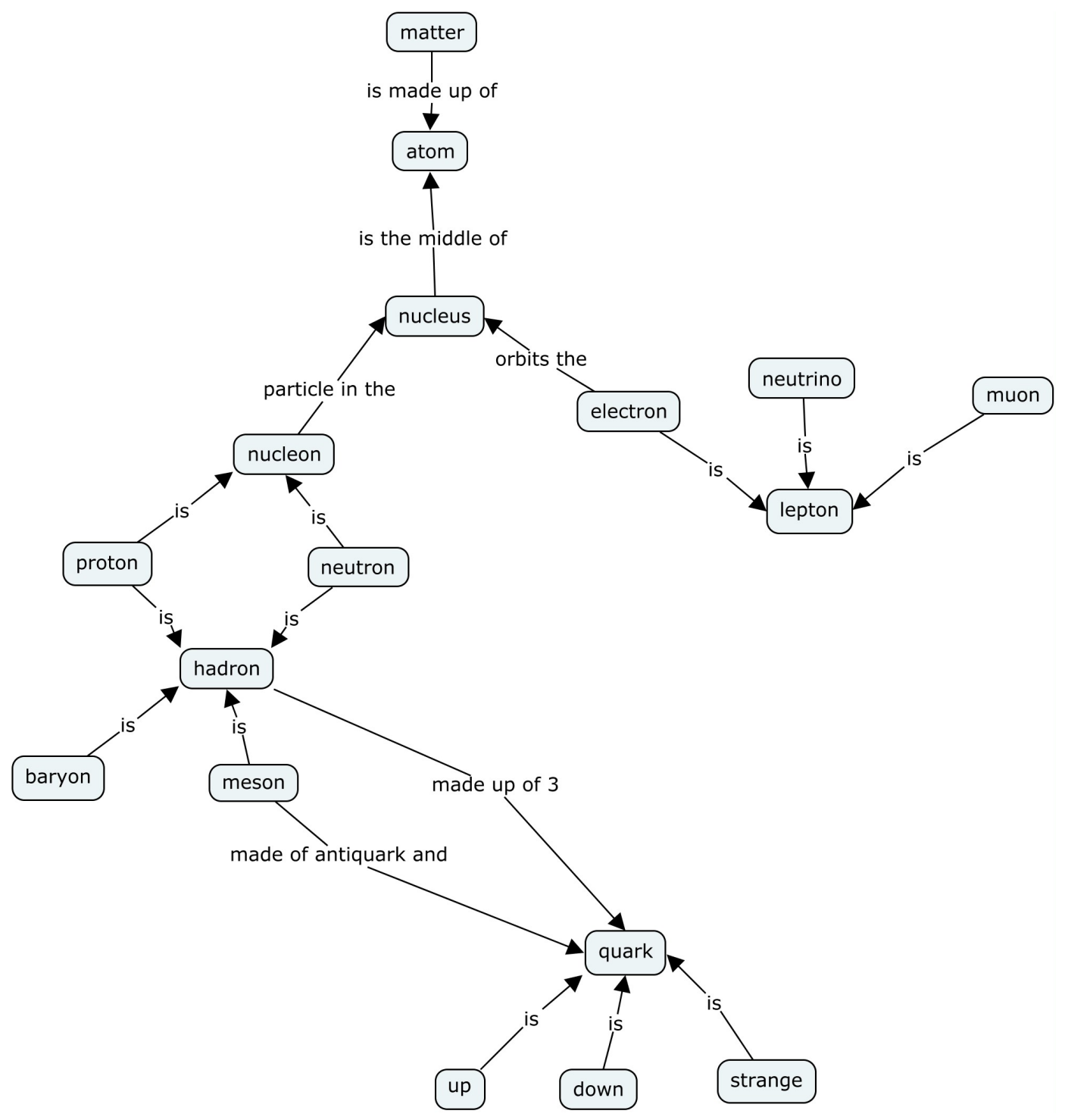

Figure 1. An example of a student's concept map

Students would have been taught earlier in their schooling (e.g. in the 11-14 age range) that the atom is an uncuttable bit, which may account for the proposition that the atom is a fundamental 
particle. The students' concept maps tended to suggest that quarks were known to be fundamental particles, but they were less clear that leptons are fundamental particles as well.

Table 2. Potential misunderstandings about leptons

\begin{tabular}{ll}
\hline Proposition & No. of instances \\
\hline Muon is a meson & 5 \\
Tau is a meson & 5 \\
Tau is a quark & 4 \\
Meson is a lepton & 1 \\
Hadron is a lepton & 1 \\
Pion is a lepton & 1 \\
Kaon is a lepton & 1 \\
Leptons have an associated neutrino & 1 \\
Muon is a quark & 1 \\
\hline Total & 20 \\
\hline
\end{tabular}

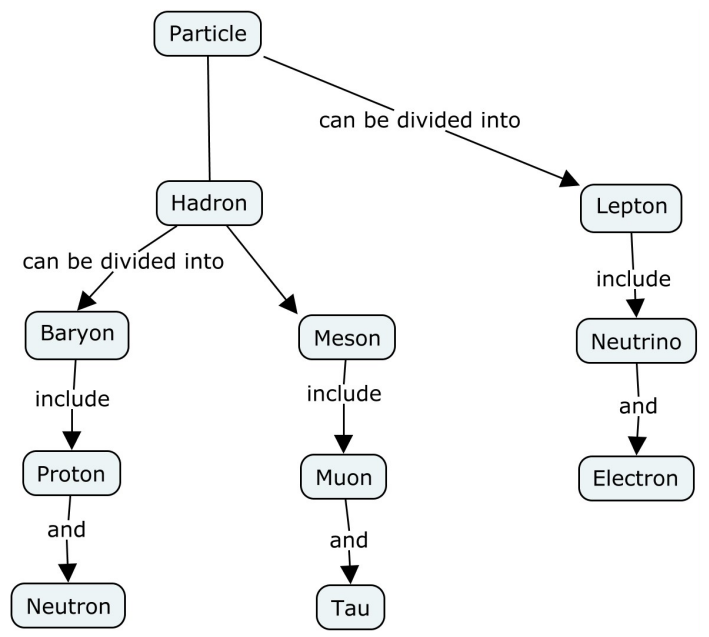

Figure 2. A concept map showing incorrect classification of muon and tau particles

\section{Recommendations}

One of the weaknesses of the study was that I sometimes needed to infer what students had meant from what they had drawn in their concept maps. In future research, it would be helpful to carry out follow-up interviews with a sample of the students in order to better understand their meaning. Compared with the study carried out by Lynch and Paterson [5] a smaller proportion of the students represented the correct structure of the atom. This may have been accounted for by the difference in research methods (since Lynch and Paterson had used multiple choice tests), and follow-up interviews may have helped me to clarify whether the A level students knew the structure of the atom in terms of protons, neutrons and electrons. Similarly, charge was rarely mentioned in the concept mapping 
exercise, and it is not possible to know from the current data whether this omission indicates a lack of knowledge, or a weakness in the method used.

It should be noted that this study did not look at the strategies for teaching that were used, so another recommendation for further research would be to investigate the effectiveness of different teaching strategies in overcoming students' misunderstandings about the topic.

I have referred to the textbook endorsed by the exam board to make some suggestions for teaching the topic at A level [11]. Firstly, I would suggest introducing the standard model at the start of the topic, since this material was omitted from the textbook. Perhaps reference to the standard model would help to support students in learning that there are two families of fundamental particles (quarks and leptons), and challenge the idea that everything is made of quarks.

Referring to the standard model may also help students to classify muons and tau particles more successfully. In the course textbook muons were introduced alongside pions and kaons because they were all discovered in the course of studying cosmic rays. It would be a good idea for teachers to explicitly address the differences between these particles, since teaching them concurrently may account for some students thinking that muons are mesons.

It is interesting to note that annihilation and pair production appeared on the same page of the textbook, which may explain why some students were unable to distinguish between them. In the textbook, annihilation was introduced in the context of PET scanning. Perhaps a different context, such as the interaction of X-rays with matter, could be used for teaching pair production.

The concept mapping technique uncovered a wealth of information about how students view the structure of the topic. I would recommend that teachers both in schools and universities consider using this method to promote discussion. It may be particularly helpful for teachers in universities whose students have learned about particle physics in high school as a means of uncovering areas about which the students are confused. Concept mapping has the potential to help teachers at all levels to build on what students already know and to address misunderstandings and misconceptions.

\section{References}

[1] Swinbank E, Physics Education 27, 87-91 (1992)

[2] Barlow R, Physics Education 27, 92-95 (1992)

[3] Novak J D and Gowin DB, Learning How to Learn Cambridge University Press, Cambridge, 1984

[4] Driver R, Making Sense of Secondary Science Routledge, London, 1994

[5] Liu X and Lesniak K M, Science Education 89, 433-450 (2005)

[5] Lynch P P and Paterson R E, Research in Science Teaching 17, 307-14 (1980)

[6] Taber K S, Research in Science Education 43, 1377-406 (2012)

[7] The Assessment and Qualifications Alliance GCE Physics A (2450) 2009 Onwards Manchester, AQA Education, 2007

[8] White R and Gunstone R, Probing Understanding London, The Falmer Press, 1992

[9] Trevillion A and Priddle G (ed), A2 Salters Horners Advanced Physics Oxford, Heinemann, 2001

[10] Gourlay H, Physics Education 52, 1-10 (2017)

[11] Breithaupt J, AQA Physics A AS: Exclusively Endorsed by AQA Cheltenham, Nelson Thornes, 2008 